\title{
System level user behavior biometrics using Fisher features and Gaussian mixture models
}

\author{
Yingbo Song \\ Allure Security Technologies Inc. \\ NewYork, NY, 10001 \\ yingbo@alluresecurity.com
}

\author{
Malek Ben Salem \\ Accenture Technology Labs \\ Arlington, VA, 22203 \\ malek.ben.salem@accenture.com
}

\author{
Shlomo Hershkop \& Salvatore J. Stolfo \\ Allure Security Technologies Inc. \\ NewYork, NY, 10001 \\ shlomo@alluresecurity.com \\ sal@alluresecurity.com
}

\begin{abstract}
We propose a machine learning-based method for biometric identification of user behavior, for the purpose of masquerade and insider threat detection. We designed a sensor that captures system-level events such as process creation, registry key changes, and file system actions. These measurements are used to represent a user's unique behavior profile, and are refined through the process of Fisher feature selection to optimize their discriminative significance. Finally, a Gaussian mixture model is trained for each user using these features. We show that this system achieves promising results for user behavior modeling and identification, and surpasses previous works in this area.
\end{abstract}

\section{INTRODUCTION}

User authentication is one of the most important topics in computer and network security research. Modern security mechanisms that aim to prevent system misuse, data leakage, and enforce security polices, are fundamentally built upon a system of trust - trust that the individuals who supplied the credentials are the same ones whom those credentials were assigned, and that the users are not overstepping the bounds of the roles their credentials represent. These are the fundamental problems of insider threat detection.

While user management is a well-understood discipline, it nevertheless remains as the main vector of actual system penetrations observed in the practice. System-level attack vectors such as software exploits, while harder to prevent, are comparably rare, and attacking the vulnerabilities inherent in the human element of the network has proven to be a more reliable method of entry in practice. These attacks do not leverage network policy shortcomings, nor do they exploit unknown software vulnerabilities; they attack the lowest hanging fruit of the network, which are often the non-security-conscious users. Spear-phishing attacks vectors, such as those used by the recent New York Times hacking incident remain a serious threat. Further, these attackers may not always originate from external sources; insider threats, where co-workers steal or otherwise misuse each other's credentials and obtain information that they should not have access to, are equally troubling.

Typically, once a user's credentials have been compromised, the incident evolves beyond the stage of defensepenetration, and into one of persistent threat, which is arguably much more difficult to detect and contain. It is within this stage that the most damage is often done to the system: backdoors are installed, files are exfiltrated, additional user accounts are compromised, and more system resources are hijacked, to be used for additional exploits. This stage is often very dynamic and can last anywhere between minutes to years, and how far the attacker reaches in this stage often determines whether the incident is a benign lesson in security discipline or a catastrophic loss to the enterprise, costing the target entity considerable financial and reputation damage.

In this study, we explore the potential of system-level user behavior biometrics, measured at the host level - a host, in this context, refers to a single workstation. The term system-level refers to the measurement perspective: we look at user behavior by quantifying changes to the operating environment in the presence of user interaction, as well as triggered events. These include registry, file, process changes, as well process creation, and more. This monitoring focuses on the persistent threat stage, after the user's credentials have already been validated, and the user has obtained access to the system. Our study monitors user-behavior during moments of known legitimate activity, then encapsulates and represents these behaviors using statistical models. These measurements are evaluated with reference to timing information as well, and are used to establish a baseline for user behavior that can then be used to test future observed actions, thus creating a notion of a biometric fingerprint for user behavior.

Our study makes several contributions to this field. First is the RUU ("are you you?") sensor, which is a logging tool that records user behavior and its effect on the operating environment. Second is the feature extraction and representation methodology, which distills the data that was captured by the sensor into statistical measurements that are optimized for behavior classification. And third, we evaluate a range of different statistical models and demonstrate that the Gaussian mixture model performs the best in this environment. Our work represents an initial study in this area, and is a subject of an ongoing research that involves not only methodology design, but also user studies as well as data collection. This paper is a summary of the key lessons observed so far in this work.

\section{BACKGROUND AND RELATED WORKS}

\section{A. Continuous Authentication}

Behavioral biometrics can provide user authentication in a non-intrusive fashion to the user. Several approaches have been proposed to authenticate users at the beginning of a user session. Most were based on modeling mouse or keystroke dynamics, either alone [1], [2], [3], [4] or in conjunction with another authentication mechanism [5]. Other behavioral biometrics work investigated the attribution of a user session to a given user once the session is completed. Goldring 
modeled process information extracted from the process table and successive window titles to profile user behavior during an entire user session [6]. Several studies modeled sequences of user command data [7], [8], [9].

However, little work focused on behavioral biometrics as active authentication mechanisms throughout the entire user session. Keystroke and mouse dynamics emerged as the main continuous authentication approaches, as they do not require any specialized equipment or additional hardware sensors. They verify computer users periodically on the basis of typing or mouse use styles. Various modeling approaches have been proposed with varying accuracy results.

Messerman et al. presented a non-intrusive continuous authentication mechanism based on free-text keystroke dynamics [10]. They used two-class modeling for profiling user keystroke dynamics behavior. Authentication of the target user is performed by scoring the user's activity against a constant number of users, but not the entire user space, to improve performance. The experiments and results were limited to the use of one application only, namely Webmail. Most users multi-task, and therefore it's important to model mouse and keystroke dynamics across various applications.

In [11], Shen, Cai and Guan proposed on a continuous authentication mechanism based solely user mouse dynamics patterns. They distinguished between two types of mouse behavior: frequent segments of mouse dynamics, which they referred to as patterns, and the less frequent segments, referred as holistic behavior. Patterns are classified as "micro-habitual" or "task-intended". The former are patterns that characterize a user's unconscious habits, such as repeatedly refreshing a screen with no real need or purpose. The task-intended patterns describe user mouse actions that are dependent on the application being used, such as opening a document from that application. They found that "patterns" are more descriptive of user behavior as they are stable features across user sessions. The same patterns emerged as discriminative features. All one-class classifiers trained using mouse activity patterns as features performed better than classifiers modeling the user's holistic behavior.

Pusara and Brodley built C5.0 decision trees on the basis of users' mouse movements within a time window of configurable size, and used the models to re-authenticate users [12]. The data was collected in a free environment, i.e. from the users' own computers. But the user sample was too limited to report generalizable results. The user mouse movements models, which were trained using data from all 11 users, achieved an average false-acceptance rate (FAR) of $1.75 \%$ and average false-rejection rate (FRR) of $0.43 \%$, but the verification time took up to 15 minutes depending on the window size.

Jagadeesan and Hsiao reported that combining keystroke and mouse dynamics reduced accuracy results, as opposed to using one of the two approaches only for continuous authentication [13]. Their experiments, however, involved only a limited set of users (5 users in each experiment.)

Although some work has reported promising results, authentication using mouse dynamics and free-test keystroke dynamics remain an immature authentication approaches. They were tested within limited and pre-defined settings (working on a specific task or interacting with one application), and therefore have not dealt with the intrinsic behavioral variability as the user interactions with various applications, or multi-tasks. We do not run our experiments in a controlled environment dependent on specific software application or hardware device. Instead, we monitor user high-level actions as they interact with their own computers and perform their daily business activities. Furthermore, our approach is less vulnerable to changes in user behavior due to physiological factors such as pain or injury, which might affect keyboard or mouse dynamics.

\section{RUU SENSOR}

This section describes the RUU sensor that was used to capture the user behavior dataset and the parameters of the user study. In this paper, we often refer to our sensor as the "Windows Sensor" to distinguish it from the Linux and Mac OSX variants that we have also developed. While the Windows sensor utilizes certain Windows-specific components to obtain the necessary measurements, the classes of measurements (e.g. file actions, process creation, etc) that we study are universal, and utilized consistently across OS platforms. The RUU user behavior measurement sensor is one of several components of our insider threat detection system. The other components are the statistical behavior modeling, and decoy-based accesstracking systems.

The Windows sensor is designed in a modular fashion, to allow multiple sensor agents to monitor specific patterns of usage on the host system. The current components include Windows Touch, File Touch, Network Touch, Process Monitor, Decoy Touch, System Log Monitor. The code base is written in .NET and is compiled to run on both 32 and 64 bit versions of windows XP, Windows 7, and Windows 8 .

The "Windows Touch" component monitors the currently active window that is in the foreground. This includes menu pop-up windows and system alert windows. We use the kernel system call "GetForegroundWindow" to get the currently active window handle, and then lookup the process ID of the process owner of the handle via the "GetWindowThreadProcessId" call. We cache the window title, and if the title has changed, we log a hashed version of it in order to be able to track search terms and title term patterns. This sensor allows us to track program usage of the active user, as users tend to multitask via active windows on their desktops.

The File Touch component registers event handlers by extending the "FileSystemWatcherEx" class for file system changes on the root file system. For any event generated, we check they type of event and depending on the level of logging, we either log every event, or potential decoy events (which are passed to the Decoy Touch Monitor).

The decoy-related component utilizes beaconing technology, watermark embedding, and native Windows accesscontrol functionality to detect unauthorized access in the persistent threat stage, post full authentication. The premise is that stolen credentials would not prevent an intruder from activating certain tripwire mechanisms, that can then be also be used to track that person. The decoy components to present to provide a scope for this work, but their use is not a factor in the results presented in this paper. 
Events that we monitor include changes to the file-access time, rename-file changes, file creation and deletion, and file security setting changes. Note that a single discrete event can trigger multiple alerts, which we filter out using a ten record filter (logging out overlapping events). Also, we filter out common background file changes to temporary and system level files. In order to decrease background noise, we also filter out high-count repeated events, based on the last hour of generated events. There are typically many background processes, all generating repeated events; we actively filter some of the identifiable ones out from the dataset.

The Network Touch module monitors open and closed events using the built in .NET call to the open port list. We use internal data structures to track changes over time. These events can be filtered out based on monitoring only the host side ports or also remote based connections. We log the local port, remote IP and port and the connection state.

Process monitor uses the .NET libraries to get a handle to the process table. For each new process ID we use the kernel functions "NtQueryInformationProcess" and "QueryFullProcessImageName" to lookup the name and path of the executable and also the parent process ID. We log newly created and deleted processes.

Decoy Touch events are triggered by the File Monitor agent. When a potential decoy file is touched, we scan new files (based on their MD5 checksum and location) for embedded watermarks and note if it is a decoy file or not. We attempt to tie the decoy-file-open event to the process that has a handle to the file, in order to differentiate between background process touches, such as backup and indexing, and userinitiated touches. The System Log monitor uses .NET library calls to monitor new system log events.

The agents can use a shared inter-process mailslot object, or internal queue list, for messages to pass log events to the central module. The central module is embedded in the host GUI for the RUU sensor to allow the users to set configurable options, and view the latest sequence of events as they happen. It also allows the user to register the local agent, and view their own data.

\section{A. RUU1 User Study}

The first iteration of the RUU user study was conducted at Columbia University in 2011, and spanned the duration of four weeks. 18 users - drawn from the University student body participated, and installed the sensor on their own computers. Only Windows sensors were used in this study. The results were collected and filtered to meet IRB standards for privacy and anonymity, and the resulting dataset was made available to the research community for the purpose of insider threat detection research. This dataset was used in this particular study. The results from this study, and related works since, help drive the next iteration of the RUU sensor design. We are currently in the process of finalizing the second RUU sensor and are preparing to begin a second round of user studies. For more details about the procedure, refer to our prior paper [14]

\section{FISHER FEATURES AND GMM}

The RUU sensor measures 18 distinct user behavior signals; these are listed later in this section. These signals are de- signed to track process creation, process window manipulation, registry actions, and file system actions. These measurements were evaluated using sliding windows of activity, conditioned on time. In our case the window size was two minutes. For a given time window, the signal measurements are considered the features for user behavior, and are treated as a biometric sample for the way that user interacts with the system. This sample is the basis by which future judgments are made on whether the behavior is normal or abnormal. Our premise is that different users will induce different behavior profiles, which will be reflected in the feature distributions observed within these windows.

Naturally, the question arises of which features are more useful for biometrics, and which are not: which can guide the design of additional behavior metrics, and which measurements have no statistical significance and should not be pursued. In fact, in our experiments, we found that performance is suboptimal when the classifiers used all of the features concurrently. This was primarily due to the fact that the features are mostly independent, given the nature of the measurement environment. For example, the number of file touches is not directly dependent on the number of registry key actions (though they may be correlated). For user identification, certain measurements will obviously be more discriminative than others in separating different classes of behavior. However, classification cannot be made on the basis of independent measurements themselves, but must be treated as a whole, as they reflect the observable output of a complex behavior process with potentially many latent variables. It is for this reason that we use a multivariate Gaussian mixture model (GMM) as our classifier. This model treats each window of user behavior as an independent and identically distributed (I.I.D) vector sample, where the feature measurements make up the vector components, i.e.:

$$
\mathbf{x}_{i}=\text { [\# of processes created, \# of registry keys added,...]. }
$$

Using features with low discriminative potential effectively induces a whitening effect on the overall distribution - artificially increasing the distribution's variance and reducing classification accuracy.

To optimize classification performance, we apply the techniques of feature selection for multi-class learning. Specifically, selection is made based on a ranking of discriminative power of each independent feature, measured using Fisher's criteria. This process is as follows: for any feature, let $\mu_{1}, \mu_{2}, \ldots, \mu_{k}$ represent the statistical means of that feature, measured over the data associated with each of the $k$ users. For example, $\mu_{i}$ is the mean of that feature measurement for the data belonging to user $i$. Let $\mu_{g}=1 / k \sum_{i=1}^{k} \mu_{k}$ represent the overall average, or grand mean, of the different classes. The scalar Fisher score is defined as:

$$
f=\frac{\sigma_{b}}{\sum_{i=1}^{k} \sigma_{i}}
$$

where $\sigma_{b}$ is known as the between-class variance:

$$
\sigma_{b}^{2}=\frac{1}{k} \sum_{i=1}^{k}\left(\mu_{i}-\mu_{g}\right)^{2},
$$

and $\sigma_{i}^{2}$ is the within-class variance. The between-class variance reflects the distance (or separability) between the different 
classes, while the within-class variance measures the variance within that particular class; a smaller variance indicates that the feature measured for a particular class is stabilized around a specific value. Similarly, if the feature's between-class variance is high, it indicates that the feature is comparably more discriminative as measurements of this feature among different classes vary greatly. The Fisher score is highest when a feature exhibits both low within-class variance and high between-class variance.

TABLE I. FISHER-SELECTED MOST DISCRIMINATIVE FEATURES FOR FIVE-MINUTE WINDOWS.

\begin{tabular}{cll}
\hline Feature & FLD Score & \\
\hline 1 & Number of unique processes & 0.0359 \\
2 & Number of registry key deletion actions & 0.0018 \\
3 & Number of create sec actions & 0.0017 \\
4 & Number of processes created & 0.0015 \\
5 & Number of files touched & 0.0013 \\
6 & Number of registry flush-key actions & 0.0012 \\
7 & Number of user touches & 0.0011 \\
8 & Number of registry key queries & 0.0011 \\
9 & Number of registry value queries & 0.0010 \\
10 & Number of processes destroyed & 0.0010 \\
11 & Number of open key actions & 0.0010 \\
12 & Number of manual search actions & 0.0009 \\
13 & Number of enumerate registry key value actions & 0.0009 \\
14 & Number of registry key creation actions & 0.0009 \\
15 & Number of window focus change actions & 0.0009 \\
16 & Number of registry key set value actions & 0.0009 \\
17 & Number of registry load key actions & 0.0009 \\
18 & Number of registry enumerate key actions & 0.0009 \\
\hline
\end{tabular}

Table I shows the list of RUU features, sorted by their Fisher scores, with the respective scores listed. For our dataset, the most discriminative feature was the number of processes run by each user; this indicates that different users within our study group not only used different numbers of applications but this behavior was also consistent across the measured time span. This property exists even after the dataset has been filtered to normalize the behavior profiles (as described below.) We note that this is a discovered property of the RUU dataset, and is not guaranteed to be consistent across all user datasets. However, this procedure allows one to generate a similar ranking given new data.

This feature ranking reflects a system level view, as captured by the RUU sensor, and is influenced by certain factors not directly related to user behavior. Given the way the Windows architecture is designed - and the lack of native support for behavior monitoring - it is often difficult to separate user actions from system actions. System actions, in this case, refers to those actions triggered by background processes that are not under the direct control of the user. Deciphering exactly how an RUU measured action was triggered, whether it was by a user command or a background task, is not straight forward, and because of this, the sensor does not have a clean look at userinfluenced actions. The actions captured by the sensor reflect both explicit user intent, such as commands to open a file, as well as tangential system actions, such as loading a series of registry keys each time a context menu is opened. Further, one process may read fewer registry keys upon loading than another - this behavior is not a reflection of user intent, but rather of system design, and ideally should not be factored into the behavior classification models. The purpose of a feature selection step in our methodology is to help determine which features are more related to user interaction, and which are more related to system implementation; the hypothesis being that the latter would be less discriminative under our Fishermotivated criteria.

Ultimately, we want to be able to detect when an intruder has accessed a system and is masquerading as a legitimate user. Therefore, in the ideal scenario, the exact same system environment would be used between two different users, and it is only the differences in the interaction that are factored. This is difficult to replicate in a user study given the fact that our users installed the sensors in their own controlled environments, so a single consistent operating environment was not available from the beginning. To help ensure that the measured features reflect more on the user and not system disparity, we normalize the data by filtering the entries based on range of processes observed. We did this by filtering the data so that we maintain only process-related entries where the processes were observed in at least $50 \%$ of the user base. This means that if a user is running several random processes that no other users are using, then those process-related events do not influence the measured features. This filter only affects data entries where process names are associated with the entry - specifically, the process-creation, unique-process, and process-destroyed features. This filtering procedure normalizes the data so that the evaluation environment is more consistent across different users, so that user interaction emerges as the dominant signal, instead of system implementation. We note that separating user actions from system actions is a very difficult challenge and the optimal way to remove this systemgenerated "background noise" is the subject of our ongoing research.

In addition to the scalar Fisher's method, we also evaluated the multivariate extension, known as Fisher Linear Discriminant (FLD). FLD is analogous to the 1-D scalar method described previously, with the key difference that, instead of ranking the features independently, we project the data vector into a lower dimensional subspace. The premise is it that a linear subspace exists, spanning the original, where the different classes can be more easily separated. The criteria for this selection is, therefore, applied, not to each individual feature, but to the method of selecting the basis vectors for the sub-space. Let $\mathbf{x}$ represent a vector measurement, then for every class $k$ :

$$
\boldsymbol{\mu}_{k}=\frac{1}{N} \sum_{n=1}^{N} \mathbf{x}_{n}^{k},
$$

where $\mathbf{x}_{i}^{k}$ represents the $i^{t h}$ vector for class $k$. The betweenclass and within-class covariance matrices are defined as:

$$
\begin{aligned}
\boldsymbol{\mu}_{g} & =\frac{1}{N} \sum_{c=1}^{C} \boldsymbol{\mu}_{c} \\
\mathbf{S}_{b} & =\frac{1}{K} \sum_{k=1}^{K}\left(\boldsymbol{\mu}_{k}-\boldsymbol{\mu}_{g}\right)\left(\boldsymbol{\mu}_{k}-\boldsymbol{\mu}_{g}\right)^{\top} \\
\mathbf{S}_{w} & =\sum_{k=1}^{K} \sum_{\mathbf{x} \in X_{k}}\left(\mathbf{x}-\boldsymbol{\mu}_{k}\right)\left(\mathbf{x}-\boldsymbol{\mu}_{k}\right)^{\top} .
\end{aligned}
$$

The optimal projection vector set $\mathbf{W}$ is defined as the one that maximizes the following criteria:

$$
F=\frac{\mathbf{W}^{\top} \mathbf{S}_{b} \mathbf{W}}{\mathbf{W}^{\top} \mathbf{S}_{w} \mathbf{W}}
$$


The above equation for $\mathbf{W}$ can be solved as a generalized eigenvalue decomposition problem. Note that, in our study, the number of user classes equals the number of features available, and therefore $\mathbf{S}_{b}$ is full rank. This is often not the case when working with FLD. In the more generalized setting, where the number of features typically greatly exceeds the number of classes, $\mathbf{S}_{b}$ would become singular. In this case, an additional subspace projection step using Principle Component Analysis is typically used, before Fishers criterion is applied.

The FLD method is the natural multivariate extension of the scalar criterion described at the beginning of the section, and we include this method in our study for the purpose of completeness. At this early stage of our work, where the feature set is still relatively small, the evaluations will show that the multivariate extension presents no performance gain. Given that the features currently represent independent measurements of different functions, attempting to project all measurements into a low-dimensional subspace may be superfluous. However, we expect the method to be useful further on when more complex and correlated measurements are available.

\section{A. Behavior modeling}

We use a Gaussian mixture model (GMM) to model the distribution of our feature set because we need an independent measurement for behavior normality, on a per user basis. This is opposed to a discriminative method, such as a standard support vector machine (SVM), which is optimized for multi-class separation, and where a second class is explicitly provided. While we evaluate biometrics methods in a multiclass setting - attempting to identify one user from another - , we cannot explicitly optimize the model for this purpose, because, in practice, there is no "intruder" class available to train on, and optimizing explicitly for second abnormal class is not a realistic approach. In practice, intruder detection will very likely be a result of anomaly detection. And therefore, a generative model such as the GMM, which can be trained on individual classes independently, is a more appropriate choice.

The GMM is among the most robust models available in the generative class of models. Most people are familiar with the K-Means algorithm; K-Means is simply a special case of the GMM, where the covariance matrices of the Gaussian submodels in K-Means are restricted to being diagonal (perfectly spherical). In GMM, the covariance matrices have no constraints, other than being full rank. This means that not only do the estimates for the model means evolve over time but so do the shapes of the ovals that conceptually represent the covariance matrices.

We use a standard construction for a multivariate Gaussian mixture model:

$$
\begin{aligned}
p(\mathbf{x} \mid \boldsymbol{\theta}) & =\frac{1}{(2 \pi)^{d / 2} \sqrt{|\boldsymbol{\Sigma}|}} \exp \left(-\frac{1}{2}(\mathbf{x}-\boldsymbol{\mu}) \boldsymbol{\Sigma}^{-1}(\mathbf{x}-\boldsymbol{\mu})^{\top}\right) \\
q(\mathbf{x} \mid \boldsymbol{\Theta}) & =\sum_{i=1}^{k} \pi_{i} p\left(\mathbf{x} \mid \boldsymbol{\theta}_{i}\right)
\end{aligned}
$$

where $\boldsymbol{\theta}_{i}=\left\{\boldsymbol{\mu}_{i}, \boldsymbol{\Sigma}_{i}\right\}$ and $\pi_{i}$ is the mixing coefficient. $q(\mathbf{x} \mid \boldsymbol{\Theta})$ is used to represent the likelihood function of the GMM. The set of parameters $\Theta$ is learned by optimizing the above equation using the Expectation Maximization algorithm. This algorithm involves solving for the expected value of $q(\mathbf{x} \mid \Theta)$ over all of the training data, taking the derivative of this function with respect to the parameters $\Theta=\{\boldsymbol{\mu}, \boldsymbol{\Sigma}, \pi\}$, setting this function to zero, and solving for these variables. This affectively provides one with the direction of the functions gradient. The parameters are updated so that they move in the direction of this gradient, and this process is repeated until no improvement is made.

For parameter estimation, we followed the standard Expectation Maximization (EM) technique; further details of the derivations are omitted. We provide the final update rules that were derived using this method. These update rules are sufficient to implement the algorithm.

Let $\tau_{n, k}^{(t)}$ represent the probability that a sample $\mathbf{x}_{n}$ belongs to the k-th sub model of the GMM at time estimate $t$. The update rules for the GMM parameters are:

$$
\begin{aligned}
\tau_{n, i}^{(t)} & =\frac{\pi_{i} N\left(\mathbf{x}_{n} \mid \boldsymbol{\mu}_{i}^{(t)}, \boldsymbol{\Sigma}_{i}^{(t)}\right)}{\sum_{j} \pi_{j} N\left(\mathbf{x}_{n} \mid \boldsymbol{\mu}_{j}^{(t)}, \boldsymbol{\Sigma}_{j}^{(t)}\right)} \\
\pi_{i}^{(t+1)} & =\frac{\sum_{n} \tau_{n, i}^{(t)}}{N} \\
\boldsymbol{\mu}_{i}^{(t+1)} & =\frac{\sum_{n} \tau_{n, i}^{(t)} \mathbf{x}_{n}}{\sum_{n} \tau_{n, i}^{(t)}} \\
\boldsymbol{\Sigma}_{i}^{(t+1)} & =\frac{\sum_{n} \tau_{n, i}^{(t)}\left(\mathbf{x}_{n}-\boldsymbol{\mu}_{i}^{(t+1)}\right)\left(\mathbf{x}_{n}-\boldsymbol{\mu}_{i}^{(t+1)}\right)^{\top}}{\sum_{n} \tau_{n, i}^{(t)}} .
\end{aligned}
$$

These parameters $\left\{\boldsymbol{\mu}_{1}, \boldsymbol{\mu}_{2}, \ldots, \boldsymbol{\Sigma}_{1}, \boldsymbol{\Sigma}_{2}, \ldots, \pi_{1}, \ldots\right\}$ are iteratively updated using the above equations until there is no improvement in the likelihood distribution $q(\mathbf{x} \mid \Theta)$.

In the evaluations section, we show that, even though the GMM is not explicitly optimized with multi-class separation in mind, for the problem of user-behavior modeling, it still outperformed the SVM-based alternatives which are optimized for two-class separation.

\section{Evaluation}

We evaluate our proposed methodology over the "RUU1" dataset, by classifying user behavior among different users. For every user, a unique behavior model is trained and segments of test behavior are then labeled by our system; comparing each sample against the models from all users, i.e. one-vsall classification. Accuracy reflects the percentage of correctly labeled instances.

During the RUU1 user study, monitoring was not continuous throughout the trial period - users were given the option to enable and disable sensor logging at any time. All of the users turned on their sensors intermittently, for periods ranging between a few minutes to a couple of hours at a time. These periods, in total, spanned between 5 days to 26 days, between different users, and captured a variety of user interactions encapsulating a range of different processes.

This section shows the evaluations for our feature extraction method and for the models. We show that, using Fishers criteria, one can reduce the number of features needed without effecting classification performance, quantifiably deriving a set 
TABLE II. AUC COMPARISONS BETWEEN GMM AND OTHER METHODS

\begin{tabular}{ccccccc}
\hline User & Uniq. Procs & Days & GMM & Parzen & oc-SVM P & oc-SVM R \\
\hline 1 & 71 & 9 & $\mathbf{0 . 6 5}$ & 0.55 & 0.64 & 0.15 \\
2 & 107 & 15 & $\mathbf{0 . 8 1}$ & 0.71 & 0.51 & 0.22 \\
3 & 57 & 12 & $\mathbf{0 . 9 1}$ & $\mathbf{0 . 9 1}$ & 0.49 & 0.36 \\
4 & 117 & 26 & 0.84 & 0.63 & $\mathbf{0 . 9 0}$ & 0.05 \\
5 & 91 & 9 & $\mathbf{0 . 8 7}$ & 0.83 & 0.14 & 0.55 \\
6 & 115 & 5 & $\mathbf{0 . 8 2}$ & 0.66 & 0.66 & 0.15 \\
7 & 52 & 12 & $\mathbf{0 . 9 1}$ & 0.90 & 0.11 & 0.61 \\
8 & 110 & 7 & 0.60 & 0.54 & 0.46 & $\mathbf{0 . 6 6}$ \\
9 & 98 & 15 & $\mathbf{0 . 8 0}$ & 0.70 & 0.58 & 0.11 \\
10 & 81 & 16 & $\mathbf{0 . 6 8}$ & 0.59 & $\mathbf{0 . 6 8}$ & 0.22 \\
11 & 77 & 10 & 0.51 & $\mathbf{0 . 6 3}$ & 0.49 & 0.28 \\
12 & 101 & 15 & $\mathbf{0 . 8 6}$ & 0.81 & 0.25 & 0.51 \\
13 & 91 & 14 & $\mathbf{0 . 8 5}$ & 0.77 & 0.30 & 0.46 \\
14 & 105 & 11 & $\mathbf{0 . 7 8}$ & 0.64 & 0.49 & 0.56 \\
15 & 107 & 9 & 0.67 & 0.67 & $\mathbf{0 . 7 7}$ & 0.13 \\
16 & 107 & 13 & $\mathbf{0 . 8 6}$ & 0.77 & 0.53 & 0.11 \\
17 & 111 & 14 & $\mathbf{0 . 8 5}$ & 0.79 & 0.26 & 0.57 \\
18 & 113 & 13 & $\mathbf{0 . 7 6}$ & 0.67 & 0.59 & 0.13 \\
\hline
\end{tabular}

of "most important features. We also show that the generative GMM works well for this classification environment, and outperforms discriminative models.

Table II shows the accuracy comparisons between the GMM model and other related classifiers. The column labeled "Uniq. Procs" show the number of unique processes observed on that user's workstation, and the "Days" column show the number of days worth of traffic collected from that user. The experiment is set up as one-vs.-all classification where each user's data was randomly split into training and testing sets, at $80 \%$ to $20 \%$ ratios. Single GMM was trained for each user using the training data. Then, each test sample was evaluated against all trained GMMs (representing all of the users) and the model with the highest probability score labeled that sample. In order to produce a single measure of accuracy over the spectrum of error/false-positive rates, we use the area-underthe-curve (AUC) metric, which is the integral of the ROC curve. A higher AUC value indicates stronger performance by that classifier. As the table shows, the GMM achieved superior performance for the majority of the users. Further, the performance was consistent across users with different numbers of processes and days measured.

This reflects one of the main problems when dealing with user behavior modeling in this approach: the data can often be sparse, with respect to user activity, and noisy with respect to mixing of user interaction and actions generated by background system processes. In short, the data is never perfect and the models should be designed appropriately. Tight fitting classifiers (such as the Parzen method), and those optimized for multi-class classification that are highly dependent on the quality of the training data (such as the SVMs) will underperform in these environments.

The Parzen method, listed in the table, is a non-parametric approach to modeling that is often used when the probability distribution is not known a priori. There is no de facto implementation of Parzens method, however the model is typically defined directly as a function of the training data and yields a generative model that - being unrestricted to a specific parametric form - is very flexible, fits tightly to the training data, and is accurate when a good sampling of training data is available. It is a popular method in machine learning and is therefore a good method to compare against in this

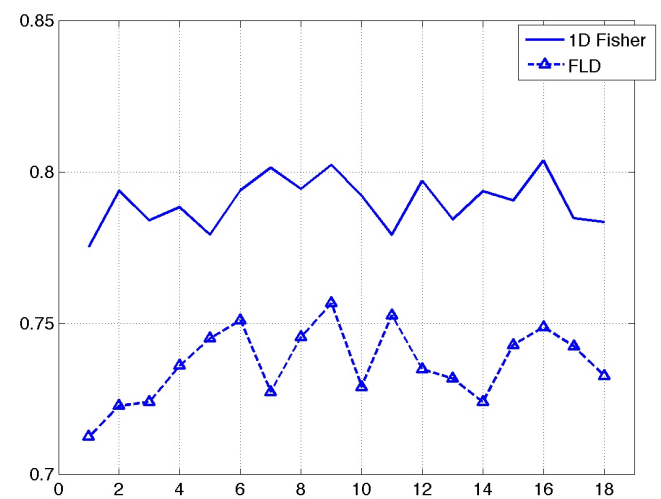

Fig. 1. Classification accuracy using Fisher-ranked features and Fisher Linear Discriminant

experiment. The support vector machine variants listed are also well known, and well understood classifiers. We used two of the most common kernels in the evaluations: the polynomial and radial basis function (RBF) kernels. The parameters of kernels were swept over during experimentation to obtain the best performing setting. The variation was the fact that we used the one-class versions of these classifiers. The one-class variations differ from the traditional two-class variations in that the hyper-plane for the SVM is optimized with respect to the distance between the support vectors and the origin (0) in the data space.

For the Parzen method, we fit a single Gaussian model per training sample. Therefore, each model is composed of $N$ Gaussian sub models where $N$ is the number of training samples for that user. The likelihood score is calculated as the product of these independent distributions.

$$
p(\mathbf{y} \mid \mathbf{X})=\frac{1}{N} \sum_{i=1}^{N} \exp \left(-\frac{1}{2}\left(\mathbf{y}-\mathbf{x}_{i}\right) \alpha \boldsymbol{I}\left(\mathbf{y}-\mathbf{x}_{i}\right)^{\top}\right),
$$

where an identity matrix, scaled by bandwidth parameter $\alpha$, is used for the covariance matrices. The optimal setting for $\alpha$ is determined through empirical testing by sweeping across a range of values. The oc-SVM columns represent one-class support vector machine models trained using the polynomial and RBF kernels, respectively. The 2nd degree polynomial kernel was used for oc-SVM P and the bandwidth parameter for the RBF kernel was optimized empirical evaluation. The construction of the support vector machines follow standard procedure, therefore we omit the details here.

As the table shows, GMM achieved superior performance in the majority of the cases, and proved to be the classifier that yielded the most consistent performance.

Next we demonstrate the utility of the Fisher's method. Figure 1 shows classification accuracy given number of Fisherselected features used. The accuracy value in the y-axis represents the average AUC score across all 18 users, as described in the previous experiment. The $\mathrm{x}$-axis represents the number of top Fisher-ranked features used in each trial: one feature means the top most discriminative feature is used, two features indicates the top two features, and so on. All reported results 


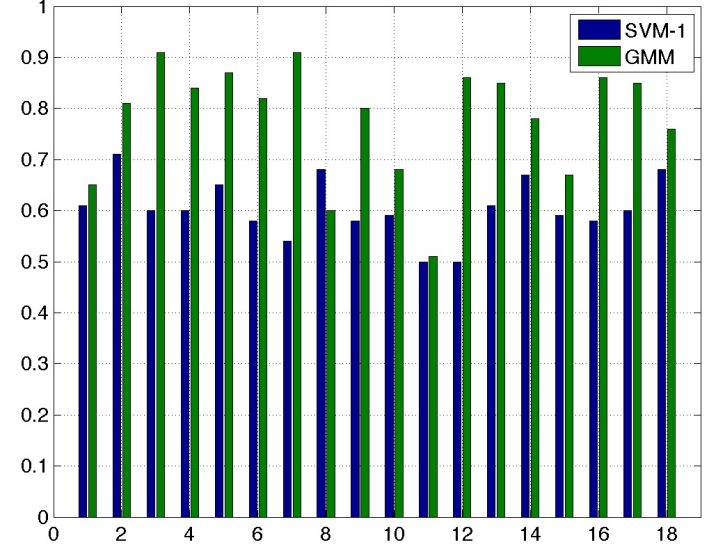

Fig. 2. Accuracy comparison with our previous method across different users. The GMM model achieves an average AUC improvement of 0.176 over the prior method (SVM-1).

are averages of five independent and randomized trials. This experiment shows that the full discriminative power of the feature set is reached after the top seven features are selected.

This provided us with insight into which features to pursue further in the next iteration of our sensor design. This result quantified the discriminative power of the features used and showed that some measurements in system behavior do not add value to the ability to identify different users from one another. This is mostly likely because these features are more influenced by system implementation and not user interaction, as previously discussed.

The fact that the lower-ranked Fisher features added no improvement in empirical testing showed to us that we needed to revisit the purpose behind their design. Given these results, the benefits of feature selection using Fisher's criterion is evident, and holds promise in this environment where were we do not yet fully understand which features best encapsulate user behavior. The fundamental reasoning behind Fisher's criteria is that the most discriminative features should exhibit relatively high between-class variance - measurements of this feature between different classes should differ highly, and small within-class variance - measurements taken from the same class should be consistent, and therefore have a smaller variance. This concept is commonly used in human biometric disciplines such as face and eye recognition, and has a natural place in behavior-based recognition as well.

The FLD subspace projection-based results are also shown in the same plot. The method was tested by projecting the data onto the top $x$ Fisher-selected basis vectors, where $x$ is shown on the $x$-axis. The FLD results are not entirely surprising, given that our sensors captured only a small set of independent user behavior features. In this case, FLD did not yield a performance benefit. The FLD method is most useful is cases where many correlated features are used such as the case of digital images. This result shows that for computer systems biometrics, the 1-D variation of Fisher's criteria should be favored ahead of the multivariate extension.
Figure 2 shows one of the key experimental results of this study, we compare our method with results from our previously published study (labeled in the figure as "SVM1") [15], [16]. This chart shows that the GMM model, with the Fisher-selected features, surpasses the prior method in all but one user instance, and achieved an average AUC improvement of $17.6 \%$ overall. This previous method was an SVM based approach using the same dataset, and one can easily see the improvements made by using the Fisher features and generative model.

\section{CONCLUSION}

In this paper, we propose new metrics for system-level user behavior biometrics. Under our method, user interactions with the system are measured with a passive behavior monitoring sensor; these signals include registry, file system, as well as process creation and deletion actions. The signals are processed through a Fisher-motivated feature selection procedure, and modeled using a multivariate Gaussian mixture model, that is then used to classify subsequent actions. We showed that, for our class of behavior measurements, the scalar Fisher feature selection was sufficient - that the multivariate FLD did not yield improved performance. We showed that a multivariate Gaussian mixture model, combined with the Fisher features, surpassed other comparable SVM-based and Parzen non-parametric approaches, and surpassed the accuracy of our previously published method by $17.6 \%$.

\section{ACKNOWLEDGEMENTS}

This research was funded by the DARPA I2O Active Authentication Program, Contract No. FA8750-12-C-0176.

\section{REFERENCES}

[1] H. Gamboa and A. Fred, "A behavioral biometric system based on human-computer interaction," pp. 381-392, Aug. 2004.

[2] A. A. E. Ahmed and I. Traore, "A new biometric technology based on mouse dynamics," IEEE Trans. Dependable Secur. Comput., vol. 4, no. 3, pp. 165-179, Jul. 2007. [Online]. Available: http://dx.doi.org/10.1109/TDSC.2007.70207

[3] C. Shen, Z. Cai, X. Guan, Y. Du, and R. Maxion, "User authentication through mouse dynamics," Information Forensics and Security, IEEE Transactions on, vol. 8, no. 1, pp. 16 -30, jan. 2013.

[4] A. Al-Khazzar and N. Savage, "Graphical authentication based on user behaviour," in Security and Cryptography (SECRYPT), Proceedings of the 2010 International Conference on, july 2010, pp. $1-4$.

[5] F. Monrose, M. K. Reiter, and S. Wetzel, "Password hardening based on keystroke dynamics," in Proceedings of the 6th ACM conference on Computer and communications security, ser. CCS ' 99. New York, NY, USA: ACM, 1999, pp. 73-82. [Online]. Available: http://doi.acm.org/10.1145/319709.319720

[6] T. Goldring, "Authenticating users by profiling behavior," in Proceedings of the ICDM Workshop on Data Mining for Computer Security, ser. DMSEC '03, 2003.

[7] M. Schonlau, W. Dumouchel, W.-H. Ju, A. F. Karr, M. Theus, and Y. Vardi, "Computer intrusion: Detecting masquerades," Statistical Science, vol. 16, pp. 58-74, 2001.

[8] R. A. Maxion and T. N. Townsend, "Masquerade detection augmented with error analysis," IEEE Transactions on Reliability, vol. 53, no. 1, pp. 124-147, 2004.

[9] M. Oka, Y. Oyama, H. Abe, and K. Kato, "Anomaly detection using layered networks based on eigen co-occurrence matrix," in Proceedings of the 7th International Symposium on Recent Advances in Intrusion Detection, ser. RAID '04, 2004, pp. 223-237. 
[10] A. Messerman, T. Mustafic, S. Camtepe, and S. Albayrak, "Continuous and non-intrusive identity verification in real-time environments based on free-text keystroke dynamics," in Biometrics (IJCB), 2011 International Joint Conference on, oct. 2011, pp. $1-8$

[11] C. Shen, Z. Cai, and X. Guan, "Continuous authentication for mouse dynamics: A pattern-growth approach," in Dependable Systems and Networks (DSN), 2012 42nd Annual IEEE/IFIP International Conference on, June 2012, pp. 1-12.

[12] M. Pusara and C. E. Brodley, "User re-authentication via mouse movements," in Proceedings of the 2004 ACM workshop on Visualization and data mining for computer security, ser. VizSEC/DMSEC '04. New York, NY, USA: ACM, 2004, pp. 1-8. [Online]. Available: http://doi.acm.org/10.1145/1029208.1029210

[13] H. Jagadeesan and M. Hsiao, "A novel approach to design of user re-authentication systems," in Biometrics: Theory, Applications, and Systems, 2009. BTAS '09. IEEE 3rd International Conference on, September 2009, pp. 1-6.

[14] M. ben Salem and S. J. Stolfo, "On the design and execution of cybersecurity user studies: Methodology, challenges, and lessons learned," in Proceedings of the Fourth Workshop on Cyber Security Experimentation and Test (CSET'11), 82011.

[15] M. Ben-Salem and S. J. Stolfo, "Modeling user search-behavior for masquerade detection," in Proceedings of the 14th International Symposium on Recent Advances in Intrusion Detection. Heidelberg: Springer, September 2011, pp. 181-200.

[16] S. J. Stolfo, M. ben Salem, and A. D. Keromytis, "Fog computing: Mitigating insider data theft attacks in the cloud," in Workshop on Research for Insider Threat (WRIT), 52012. 